\title{
Mean platelet volume and platelet distribution width levels in patients with mild psoriasis vulgaris with metabolic syndrome
}

\author{
Selma Korkmaz \\ Department of Dermatology, Faculty of Medicine, Süleyman Demirel University, Isparta, Turkey \\ Adv Dermatol Allergol 2018; XXXV (4): 367-371 \\ DOI: https://doi.org/10.5114/ada.2017.71285
}

\begin{abstract}
Introduction: Psoriasis vulgaris (PV) is a common inflammatory skin disease characterized by incomplete differentiation and hyperproliferation of epidermal keratinocytes. Platelets play a role in inflammatory reactions and the immune response and may be activated by a variety of stimulants. Studies in recent years have shown increased incidence of metabolic syndrome (MetS) in psoriasis patients. However, the mean platelet volume (MPV) and platelet distribution width (PDW) values have not been assessed in PV accompanied by MetS.

Aim: To evaluate MPV and PDW levels and their association with metabolic parameters in the presence of MetS in PV patients.

Material and methods: A total of 76 patients with mild PV, including 38 with MetS and 38 without MetS, 38 patients with MetS without PV and 35 healthy individuals were enrolled in the study. The number of platelets, MPV, PDW, fasting blood glucose, urea, creatinine, total cholesterol, high-density lipoprotein cholesterol (HDL-C), low-density lipoprotein cholesterol (LDL-C), triglycerides, thyroid stimulating hormone, free triiodothyronine, and the free thyroxine levels were evaluated.

Results: Mean platelet volume and PDW levels were significantly higher in the mild PV with MetS group compared to the mild PV without MetS group, MetS without PV patients and the healthy control group ( $p<0.05$, for all). There was no significant difference between the mild PV without MetS group and the healthy control group in terms of MPV and PDW levels ( $p>0.05$, for all). There was no significant difference between the groups with MetS and without MetS in terms of the psoriasis area and severity index (PASI) $(p>0.05)$. The PASI was not correlated with MPV and PDW. In addition, metabolic parameters were not correlated with MPV and PDW in mild PV patients. Conclusions: Mean platelet volume and PDW levels showing platelet activation increase significantly in the presence of MetS in patients with mild PV.
\end{abstract}

Key words: metabolic syndrome, psoriasis vulgaris, mean platelet volume, platelet distribution width.

\section{Introduction}

Psoriasis vulgaris (PV) is a common inflammatory skin disease characterized by incomplete differentiation and hyperproliferation of epidermal keratinocytes. In the pathogenesis of the disease, it is proposed that platelets play a role in addition to other inflammatory cells. Activated platelets increase migration of leukocytes into the skin, and this is thought to contribute to increasing the release of inflammatory cytokines from the skin [1-4]. Platelets play a role in inflammatory reactions and the immune response and may be activated by a variety of stimulants. Despite its potential contribution to atherothrombosis, measuring platelet activity takes a long time, requires special centers and is costly. The most commonly used alternatives to measure platelet activation and the easiest measured parameters are mean platelet volume (MPV) and platelet distribution width (PDW) [5]. Large platelets are more active compared to small platelets, and contain higher levels of procoagulant proteins $[6,7]$. It has been shown that MPV increases in cardiovascular diseases, peripheral diseases and diabetes mellitus [8-10]. In PV those with moderate and severe disease activity have increased MPV values and these show a positive correlation with the disease activity. However, in those with mild disease activity, no change in MPV values identified [11].

Glucose intolerance, hypertension, hypertriglyceridemia, hyperinsulinemia, low HDL, and high triglyceride levels are seen in metabolic syndrome (MetS). Studies in recent years have shown increased incidence of MetS in

Address for correspondence: Dr. Selma Korkmaz, Department of Dermatology, Faculty of Medicine, Süleyman Demirel University, 32040 Isparta, Turkey, phone: +90 5063567227, e-mail: selmakorkmaz35@gmail.com

Received: 16.05.2017, accepted: 30.05.2017. 
psoriasis patients [12]. However, the MPV and PDW values have not been assessed in PV accompanied by MetS.

\section{Aim}

This study aimed to assess the MPV and PDW levels in mild severity PV patients with and without MetS and the correlation between these levels and metabolic parameters.

\section{Material and methods}

This study received permission from Trakya University, Faculty of Medicine Ethics Committee. Patient data were collected retrospectively.

\section{Selection of cases for the study}

The study included 38 PV patients without MetS (mean age: $43 \pm 9.3$ years; 22 males, 16 females), $38 \mathrm{PV}$ patients with MetS (mean age: $44.2 \pm 10.1$ years; 18 males, 20 females), 38 MetS patients without PV (mean age: $45.8 \pm 9.7$ years; 19 males 19 females) and 35 healthy volunteers (mean age: $42 \pm 10.4$ years; 18 males 17 females) applying to Trakya University Dermatology and Venereal Disease clinic. PV diagnosis was made after clinical and/ or histopathological investigations. Those with dermatological disease other than PV; cigarette/alcohol use; systemic disease such as cardiac, renal or hepatic diseases; diabetes mellitus; diseases that may cause inflammation (e.g., acute infectious diseases, malignancy, inflammatory rheumatismal diseases); pregnancy; hematological disease (e.g., thrombocytopenia, hemoglobinopathy); and any history of medication use within the last month were excluded from the study. The gender, age, age at disease onset and disease duration of patients were recorded. Dermatological examination was performed by a single dermatologist and the psoriasis area and severity index (PASI) was calculated [13]. Patients with severe psoriasis forms such as psoriatic arthritis, erythrodermic and pustular psoriasis were not included in the study. Patients with body surface area involvement of more than $10 \%$ were removed from the study. MetS diagnosis for those with and without PV was made according to the modified NCEP ATP-III criteria [14].

\section{Anthropometric and blood pressure measurements}

All participants had body weight $(\mathrm{kg})$ and height $(\mathrm{m})$ measured. Body mass index (BMI, $\left.\mathrm{kg} / \mathrm{m}^{2}\right)$ was calculated from the ratio of body weight $(\mathrm{kg})$ to the square of height $(\mathrm{m})$. Waist circumference $(\mathrm{cm})$ was measured with tape passing both the superior iliac crest and the umbilicus. All subjects had systolic blood pressure (SBP) and diastolic blood pressure (DBP) measured after 30 min without drinking tea or smoking and 5 min rest while sitting down with a mercury sphygmomanometer used on both arms.

\section{Biochemical tests}

After $8 \mathrm{~h}$ fasting, morning blood samples were taken to measure fasting blood glucose (FBG), urea, creatinine, total cholesterol, HDL-C, LDL-C, TG, thyroid stimulating hormone (TSH), free triiodothyronine (fT3) and free thyroxine (fT4) on a Cobas Integra 800 model autoanalyzer (Roche Diagnostics, Germany). The patient and control groups had MPV, PDW and platelet count levels measured in venous blood samples placed in EDTA tubes using a Sysmex XE-2100 with the flow cytometry method (Sysmex Corporation, Kobe, Japan).

\section{Statistical analysis}

Continuous variables were checked for normal distribution with the Shapiro-Wilk test. For comparison of more than two independent groups, the Kruskal-Wallis test and Dunn multiple comparison tests were used. Variables with normal distribution were compared in 2 independent groups with Student's $t$ test (age), while variables without normal distribution were compared with the Bonferroni corrected Mann-Whitney $U$ test. For comparison of categorical variables, the $\chi^{2}$ test was used (gender). The correlation between disease severity in PV patients and hematologic and metabolic parameters was assessed with Spearman correlation analysis. Statistical analysis was carried out using the SPSS for Windows version 22.0 program and $p<0.05$ was accepted as statistically significant.

\section{Results}

The demographic, clinical and biochemical characteristics of the study groups are shown in Table 1. There was no difference between the groups in terms of age and gender ( $p>0.05$ for all).

In the metS group without PV the BMI and waist circumference were higher compared to the other groups ( $p<0.05$ for all). PV patients with MetS had higher TG and FBG compared to healthy volunteers and HDL levels were low. In the MetS group without PV, the HDL-C levels were significantly low compared to the PV with MetS group ( $p=0.046)$. There was no difference between the groups in terms of platelet count ( $p>0.05$ for all). MPV and PDW were significantly higher in the PV with MetS group compared to other groups ( $p<0.05$ for all). Though PDW was high in the PV without MetS group compared to the MetS group and healthy volunteers, this was not statistically significant ( $p=0.065$ and $p=0.160$, respectively). The MPV was higher in the PV without MetS group compared to healthy volunteers $(p=0.041)$.

The mean PASI value in the PV with MetS group was $2.51 \pm 1.90$, while in the PV without MetS group it was $3.04 \pm 2.18$. There was no difference between PV groups with and without MetS in terms of PASI $(p=0.411)$. No correlation was identified between MPV and PDW in PV 
Table 1. Clinical and laboratory characteristics of groups

\begin{tabular}{|c|c|c|c|c|c|c|c|c|c|c|}
\hline Parameter & $\begin{array}{c}\mathrm{P} \\
(n=38)\end{array}$ & $\begin{array}{c}\text { PM } \\
(n=38)\end{array}$ & $\begin{array}{c}\text { HC } \\
(n=35)\end{array}$ & $\begin{array}{c}M \\
(n=38)\end{array}$ & $\begin{array}{c}\text { P-PM } \\
P \text {-value }\end{array}$ & $\begin{array}{c}\text { P-HC } \\
P \text {-value }\end{array}$ & $\begin{array}{c}\mathrm{P}-\mathrm{M} \\
P \text {-value }\end{array}$ & $\begin{array}{l}\text { PM-HC } \\
P \text {-value }\end{array}$ & $\begin{array}{l}\text { PM-M } \\
P \text {-value }\end{array}$ & $\begin{array}{c}M-\mathrm{HC} \\
P \text {-value }\end{array}$ \\
\hline Age [years] & $43 \pm 9.3$ & $44.2 \pm 10.1$ & $42 \pm 10.4$ & $45.8 \pm 9.7$ & 0.274 & 0.392 & 0.112 & 0.385 & 0.651 & 0.090 \\
\hline Gender (M/F) & $22 / 16$ & $18 / 20$ & $18 / 17$ & $19 / 19$ & 0.342 & 0.174 & 0.664 & 0.445 & 0.224 & 0.124 \\
\hline $\mathrm{SBP}[\mathrm{mm} \mathrm{Hg}]$ & $120 \pm 15$ & $120 \pm 20$ & $120 \pm 10$ & $120 \pm 10$ & 0.384 & 0.063 & 0.050 & 0.010 & 0.040 & $<0.001$ \\
\hline $\mathrm{DBP}[\mathrm{mm} \mathrm{Hg}]$ & $80 \pm 10$ & $70 \pm 10$ & $70 \pm 20$ & $80 \pm 20$ & 0.968 & 0.063 & 0.056 & 0.051 & 0.060 & $<0.001$ \\
\hline BMI $\left[\mathrm{kg} / \mathrm{m}^{2}\right]$ & $28 \pm 4.2$ & $29.9 \pm 6.6$ & $25 \pm 5.4$ & $34 \pm 9.3$ & 0.020 & 0.039 & $<0.001$ & $<0.001$ & 0.019 & $<0.001$ \\
\hline$W C[\mathrm{~cm}]$ & $93 \pm 19$ & $103 \pm 13$ & $92 \pm 15$ & $115 \pm 27$ & 0.034 & 0.096 & $<0.001$ & $<0.001$ & 0.050 & $<0.001$ \\
\hline \multicolumn{11}{|l|}{ Laboratory findings: } \\
\hline $\mathrm{HDL}-\mathrm{C}[\mathrm{mg} / \mathrm{dl}]$ & $48 \pm 10$ & $43 \pm 20$ & $49 \pm 17$ & $39 \pm 15$ & 0.328 & 0.163 & 0.040 & 0.046 & 0.046 & $<0.001$ \\
\hline LDL-C [mg/dl] & $129 \pm 36$ & $137.5 \pm 45$ & $110 \pm 38$ & $131 \pm 41$ & 0.075 & 0.043 & 0.867 & $<0.001$ & 0.066 & 0.038 \\
\hline $\mathrm{TG}[\mathrm{mg} / \mathrm{dl}]$ & $99 \pm 50$ & $190.5 \pm 144$ & $95 \pm 50$ & $181 \pm 86$ & 0.010 & 0.272 & $<0.001$ & $<0.001$ & 0.349 & $<0.001$ \\
\hline FBG $[\mathrm{mg} / \mathrm{dl}]$ & $89 \pm 12$ & $103 \pm 28$ & $87 \pm 8$ & $107 \pm 31$ & $<0.001$ & 0.036 & $<0.001$ & $<0.001$ & 0.301 & $<0.001$ \\
\hline Platelet $\left[\times 10^{3} / \mu \mathrm{l}\right]$ & $274 \pm 100.5$ & $276.5 \pm 80$ & $263 \pm 104$ & $277 \pm 64.5$ & 0.800 & 0.588 & 0.974 & 0.373 & 0.741 & 0.525 \\
\hline MPV [fl] & $10.2 \pm 0.9$ & $10.7 \pm 1.1$ & $10 \pm 1.7$ & $9.8 \pm 1.7$ & 0.036 & 0.243 & 0.089 & 0.008 & 0.010 & 0.455 \\
\hline PDW [fl] & $12.1 \pm 1.8$ & $13.3 \pm 1.9$ & $11.6 \pm 2.7$ & $11.1 \pm 3.0$ & 0.008 & 0.160 & 0.065 & 0.002 & $<0.001$ & 0.535 \\
\hline
\end{tabular}

Data are given as mean + SD and median $\pm I O R$. P-psoriasis group, PM - psoriasis group with metabolic syndrome, HC-healthy control group, $M-$ metabolic syndrome group, SBP-systolic blood pressure, DBP - diastolic blood pressure, BMI-body mass index, HDL-C-high-density lipoprotein cholesterol, $L D L$ - $C$ - low-density lipoprotein cholesterol, TG - triglyceride, MPV - mean platelet volume, PDW-platelet distribution width, FBG - fasting blood glucose, WC - waist circumference.

Table 2. Correlations between variables in psoriasis patients

\begin{tabular}{|c|c|c|c|c|c|c|c|c|c|}
\hline & PDW & PASI & BMI & WC & SBP & DBP & FBG & TG & HDL-C \\
\hline \multicolumn{10}{|l|}{ MPV } \\
\hline $\mathrm{R}$ & 0.933 & 0.038 & 0.217 & 0.040 & -0.101 & -0.012 & -0.002 & 0.231 & 0.009 \\
\hline $\mathrm{P}$ & $<0.001$ & 0.796 & 0.157 & 0.788 & 0.496 & 0.934 & 0.988 & 0.070 & 0.946 \\
\hline \multicolumn{10}{|c|}{ PDW } \\
\hline$R$ & & 0.059 & -0.063 & -0.093 & -0.179 & -0.110 & 0.040 & 0.200 & -0.004 \\
\hline $\mathrm{P}$ & & 0.692 & 0.672 & 0.532 & 0.225 & 0.456 & 0.741 & 0.119 & 0.973 \\
\hline \multicolumn{10}{|l|}{ PASI } \\
\hline$R$ & & & -0.102 & 0.146 & -0.057 & 0.108 & -0.152 & -0.144 & -0.039 \\
\hline$P$ & & & 0.480 & 0.315 & 0.696 & 0.457 & 0.291 & 0.317 & 0.789 \\
\hline \multicolumn{10}{|l|}{ BMI } \\
\hline$R$ & & & & 0.737 & 0.304 & 0.361 & 0.217 & 0.130 & 0.116 \\
\hline$P$ & & & & $<0.001$ & 0.032 & 0.010 & 0.131 & 0.369 & 0.421 \\
\hline \multicolumn{10}{|l|}{ WC } \\
\hline$R$ & & & & & 0.356 & 0.414 & 0.163 & 0.093 & -0.022 \\
\hline$P$ & & & & & 0.012 & 0.003 & 0.264 & 0.527 & 0.879 \\
\hline \multicolumn{10}{|l|}{ SBP } \\
\hline$R$ & & & & & & 0.683 & -0.002 & -0.052 & -0.007 \\
\hline $\mathrm{P}$ & & & & & & $<0.001$ & 0.991 & 0.719 & 0.962 \\
\hline \multicolumn{10}{|l|}{ DBP } \\
\hline$R$ & & & & & & & -0.204 & -0.102 & -0.029 \\
\hline $\mathrm{P}$ & & & & & & & 0.155 & 0.479 & 0.841 \\
\hline \multicolumn{10}{|l|}{ FBG } \\
\hline$R$ & & & & & & & & 0.152 & 0.046 \\
\hline$P$ & & & & & & & & 0.221 & 0.718 \\
\hline \multicolumn{10}{|l|}{ TG } \\
\hline$R$ & & & & & & & & & -0.286 \\
\hline$P$ & & & & & & & & & 0.022 \\
\hline
\end{tabular}

MPV - mean platelet volume, PDW - platelet distribution width, BMI - body mass index, SBP-systolic blood pressure, DBP - diastolic blood pressure, FBG - fasting blood glucose, TG - triglyceride, HDL-C - high-density lipoprotein cholesterol, PASI - psoriasis area and severity index, WC - waist circumference. 
patients with metabolic parameters and disease severity (Table 2).

\section{Discussion}

To the best of our knowledge, this study is the first to assess MPV and PDW in PV accompanied by MetS. This study demonstrated that MPV and PDW levels were high in mild severity PV patients with MetS compared to PV patients without MetS.

High MPV means that the platelet size is large. Large platelets are younger, denser and more active. As a result high MPV increases the risk of vascular complications $[15,16]$. During activation of platelets, MPV and PDW increase [17]. Metabolic syndrome is an independent risk factor for cardiovascular disease [18]. Studies assessing MPV levels in MetS have produced contradictory results. Farah and Khamisy-Farah found that MPV levels were higher in those with MetS compared to those without MetS [19]. However, Kutlucan et al. did not identify a difference between obese individuals with and without MetS in terms of MPV [20]. Additionally, Shah et al. in a study of 13,021 individuals found no difference in MPV levels between non-diabetic MetS patients and healthy controls, while MPV levels were significantly high in diabetic MetS patients [21]. This study did not identify a difference in MPV and PDW levels between individuals with MetS and healthy controls ( $p<0.455$ and $p=0.535$, respectively). A correlation was identified between MPV and the carotid intima media thickness, which is a marker of the increase in subclinical atherosclerosis, in patients with MetS [22]. In line with these results, while no variation is expected in MPV levels in the early period among those with MetS, it leads to the consideration that in successive periods the marker of atherosclerotic change of MPV may increase, especially with the addition of diabetes mellitus.

Psoriasis vulgaris is a skin disease progressing with chronic inflammation [2]. In a variety of studies, the MPV and PDW of PV patients have been assessed. Canpolat et al. identified an MPV increase in psoriasis patients and those with psoriatic arthritis, and there was a positive correlation between disease activity and MPV [23]. Kim et al. in a study of 176 psoriasis patients identified increased MPV levels in psoriasis patients and found that MPV levels decreased after treatment [24]. Saleh et al. identified high MPV levels in those with moderate and severe psoriasis compared to those with mild psoriasis and healthy controls and found a positive correlation between disease activity and MPV [11]. In this study only PV patients with mild severity disease activity were included. There was no significant difference in the MPV and PDW between mild PV patients without MetS and healthy volunteers $(p=0.243)$. Additionally, no correlation was found between disease activity and MPV and PDW. This may be due to the study only including PV patients with mild severity disease. As psoriasis disease activity increases, inflammation increases. Platelets play an important role in inflammatory reactions and immune response. Platelets store a variety of proinflammatory mediators such as serotonin, cytokines and chemokines. Of these, CXCL8 and IL-1 $\beta$ levels are shown to increase with psoriasis disease [25].

Metabolic syndrome is a problem commonly observed with psoriasis disease $[12,26]$. In psoriasis patients with MetS, MPV and PDW have not been previously assessed. This study revealed that MPV and PDW levels were high in mild severity PV patients with MetS compared to healthy controls, PV patients without MetS and participants only with MetS. These findings lead to the consideration that even if disease activity is mild, PV with MetS may increase the risk of development of atherosclerotic diseases. To reduce cardiovascular morbidity and mortality in these patients monitoring MPV and PDW may be important.

\section{Conclusions}

In patients with mild severity PV and MetS who do not have diabetes mellitus MPV and PDW levels may not display any significant change, but the combination of the two diseases increases MPV and PDW levels. This situation indicates that when both diseases are combined, monitoring MPV and PDW may be important in terms of atherosclerotic disease risk. There is a need for comprehensive studies on this topic.

\section{Conflict of interest}

The author declares no conflict of interest.

\section{References}

1. Kasperska-Zajac A, Brzoza Z, Rogala B. Platelet function in cutaneous diseases. Platelets 2008; 19: 317-21.

2. Boehncke WH. Etiology and pathogenesis of psoriasis. Rheum Dis Clin North Am 2015; 41: 665-75.

3. Kılıç S, Reşorlu H, Işık S, et al. Association between mean platelet volume and disease severity in patients with psoriasis and psoriatic arthritis. Adv Dermatol Allergol 2017; 34: 126-30.

4. Kwiek B, Narbutt J, Sysa-Jędrzejowska A, et al. Long-term treatment of chronic plaque psoriasis with biological drugs can control platelet activation: targeting the bridge between inflammation and atherothrombosis. Adv Dermatol Allergol 2017; 34: 131-7.

5. Leader A, Pereg D, Lishner M. Are platelet volume indices of clinical use? A multidisciplinary review. Ann Med 2012; 44: 805-16.

6. Thompson CB, Jakubowski JA, Quinn PG, et al. Platelet size and age determine platelet function independently. Blood 1984; 63: 1372-5.

7. Pathansali R, Smith NM, Bath PM. Prothrombotic megakaryocyte and platelet changes in hypertension are reversed following treatment: a pilot study. Platelets 2001; 12: 144-9. 
8. Berger JS, Eraso LH, Xie D, et al. Mean platelet volume and prevalence of peripheral artery disease, the National Health and Nutrition Examination Survey, 1999-2004. Atherosclerosis 2010; 213: 586-91.

9. Abalı G, Akpınar O, Söylemez N. Correlation of the coronary severity scores and mean platelet volume in diabetes mellitus. Adv Ther 2014; 31: 140-8.

10. Sansanayudh N, Numthavaj P, Muntham D, et al. Prognostic effect of mean platelet volume in patients with coronary artery disease. A systematic review and meta-analysis. Thromb Haemost 2015; 114: 1299-309.

11. Saleh HM, Attia EA, Onsy AM, et al. Platelet activation: a link between psoriasis per se and subclinical atherosclerosis: a case-control study. Br J Dermatol 2013; 169: 68-75.

12. Armstrong AW, Harskamp CT, Armstrong EJ. Psoriasis and metabolic syndrome: a systematic review and meta-analysis of observational studies. J Am Acad Dermatol 2013; 68: 654-62.

13. Ku SH, Kwon WJ, Cho EB, et al. The association between psoriasis area and severity index and cardiovascular risk factor in Korean psoriasis patients. Ann Dermatol 2016; 28: 360-3.

14. Heng D, Ma S, Lee JJ, et al. Modification of the NCEP ATP III definitions of the metabolic syndrome for use in Asians identifies individuals at risk of ischemic heart disease. Atherosclerosis 2006; 186: 367-73.

15. Ulutas KT, Dokuyucu R, Sefil F, et al. Evaluation of mean platelet volume in patients with type 2 diabetes mellitus and blood glucose regulation: a marker for atherosclerosis? Int J Clin Exp Med 2014; 7: 955-61.

16. Kodiatte TA, Manikyam UK, Rao SB, et al. Mean platelet volume in type 2 diabetes mellitus. J Lab Physicians 2012; 4: 5-9.

17. Vagdatli E, Gounari E, Lazaridou E, et al. Platelet distribution width: a simple, practical and specific marker of activation of coagulation. Hippokratia 2010; 14: 28-32.

18. Wang Y, Yu Q, Chen Y, Cao F. Pathophysiology and therapeutics of cardiovascular disease in metabolic syndrome. Curr Pharm Des 2013; 19: 4799-805.

19. Farah R, Khamisy-Farah R. Significance of MPV, RDW with the presence and severity of metabolic syndrome. Exp Clin Endocrinol Diabetes 2015; 123: 567-70.

20. Kutlucan A, Bulur S, Kr S, et al. The relationship between mean platelet volume with metabolic syndrome in obese individuals. Blood Coagul Fibrinolysis 2012; 23: 388-90.

21. Shah B, Sha D, Xie D, et al. The relationship between diabetes, metabolic syndrome, and platelet activity as measured by mean platelet volume: the National Health And Nutrition Examination Survey, 1999-2004. Diabetes Care 2012; 35: 1074-8.

22. Gu Lcan AR, Karakaş MS, Akdemir B, et al. Relation between mean platelet volume and subclinical atherosclerosis in patients with metabolic syndrome. Turk Kardiyol Dern Ars 2014; 42: 22-8.

23. Canpolat F, Akpinar H, Eskioğlu F. Mean platelet volume in psoriasis and psoriatic arthritis. Clin Rheumatol 2010; 29: 325-8.

24. Kim DS, Lee J, Kim SH, et al. Mean platelet volume is elevated in patients with psoriasis vulgaris. Yonsei Med J 2015; 56: 712-8.

25. Pietrzak AT, Zalewska A, Chodorowska G, et al. Cytokines and anticytokines in psoriasis. Clin Chim Acta 2008; 394: 7-21.

26. Voiculescu VM, Lupu M, Papagheorghe L, et al. Psoriasis and metabolic syndrome: scientific evidence and therapeutic implications. J Med Life 2014; 7: 468-71. 